\title{
Local electronic density of states of a semiconducting carbon nanotube interface
}

\author{
Hajin Kim, Jhinhwan Lee, Sungjun Lee, and Young Kuk \\ School of Physics and Center for Science in Nanometer Scale, Seoul National University, Seoul, 151-742, Korea \\ Ji-Yong Park \\ Department of Physics, Ajou University, Suwon, 442-749 Korea \\ Se-Jong Kahng \\ Department of Physics, Korea University, Seoul, 136-713, Korea
}

(Received 29 December 2004; published 2 June 2005)

\begin{abstract}
The local electronic structure of semiconducting single-wall carbon nanotubes was studied with scanning tunneling microscopy. We performed scanning tunneling spectroscopy measurement at selected locations on the center axis of carbon nanotubes, acquiring a map of the electronic density of states. Spatial oscillation was observed in the electronic density of states with the period of atomic lattice. Defect induced interface states were found at the junctions of the two semiconducting nanotubes, which are well-understood in analogy with the interface states of bulk semiconductor heterostructures. The electronic leak of the van Hove singularity peaks was observed across the junction, due to inefficient charge screening in a one-dimensional structure.
\end{abstract}

DOI: 10.1103/PhysRevB.71.235402

PACS number(s): 68.37.Ef, 81.07.De

\section{INTRODUCTION}

Carbon nanotubes (CNTs) are promising materials, with many possible applications due to their excellent material characteristics. ${ }^{1,2}$ The electronic conductivity and the thermal conductivity of CNTs can be as good as or even better than those of noble metals. The elastic modulus of CNTs is an order of magnitude higher than that of steel alloys. CNTs are electronically one-dimensional (1D) and either metallic or semiconducting, depending on their cylindrical wrapping structures and diameters. It is known that one can control the electronic properties of CNTs by introducing defects, ${ }^{3,4}$ molecules, ${ }^{5}$ and chemical doping. ${ }^{6,7}$ It has been proposed that the junction structures of CNTs might be useful in constructing electronic devices. ${ }^{3,4,7,8}$ For example, optoelectronic devices such as light-emitting diodes can be made by using semiconducting-semiconducting (S-S) CNT junctions with different band gaps. It is known that CNT junctions can be made by fusing two neighboring CNTs with a high electric field. ${ }^{9}$

In bulk semiconductors, there often exist defect-induced surfaces and interface electronic states in the forbidden energy gap between the valence and conduction bands. ${ }^{10}$ In many metal-semiconductor (M-S) and S-S junctions, the electronic characteristics are strongly governed by the surface states and interface states. Examples of this are the Fermi level pinning and the pinning of Schottky barrier height. When the density of interface states is much greater than the density of bulk dopant states, the Fermi level and Schottky barrier height do not change by very much with increased doping density, resulting in the pinning of the Schottky barrier height.

It was reported that there exist characteristic end states at the end of single wall CNTs. ${ }^{11}$ The end states are in the energy gaps of semiconducting CNTs, implying that the end states are a 1D analogy with the surface states of bulk semiconductors. It was reported that interface states were ob- served below the Fermi energy level in metal-metal (M-M) CNT junctions. ${ }^{4}$ The decay lengths of the interface states were reported to be $\sim 1 \mathrm{~nm}$. At M-S CNT junctions, on the other hand, no interface state has been experimentally manifested. Similarly, no interface state was reported at the kink of an M-S CNT junction. ${ }^{12}$ One may be curious if there is any interface state in a S-S CNT junction.

In this paper, we report on the electronic structure of an $\mathrm{S}-\mathrm{S}$ CNT junction prepared on the $\mathrm{Au}(111)$ surface. We obtained maps of the local electronic density of states (LDOS) using scanning tunneling spectroscopy (STS). We found interface states at S-S CNT junctions. The interface states are in the energy gaps of semiconducting CNTs. An interface state shows unequal decay lengths of the two CNTs, and the decay lengths were measured to be a few nanometers, which are longer than that those of an M-M CNT junction.

\section{EXPERIMENTS}

Single-wall CNTs made with the laser ablation method were sonicated for $>1 \mathrm{~h}$ in dichloroethane to produce a uniform colloidal suspension, which remains intact for months after settling down. We cleaned an $\mathrm{Au}(111)$ sample by repeated cycles of sputtering and annealing in an ultrahigh vacuum (UHV) chamber. After that, a droplet of the CNTs suspension was dispersed on the $\mathrm{Au}(111)$ surface with a glass pipet. To avoid contamination in an ambient environment, we performed this procedure in a glove box filled with pure Ar gas, connected to the UHV chamber through a load-lock system. When the solvent in the droplet was completely dried on the $\mathrm{Au}(111)$ surface, the sample of CNTs on $\mathrm{Au}(111)$ was put back into the UHV chamber with a scanning tunneling microscope (STM). In the UHV chamber, the sample was heated again to $\sim 150{ }^{\circ} \mathrm{C}$ to remove the residual solvent. Figure 1 shows typical STM images with CNTs on $\mathrm{Au}(111)$. We observed isolated and bundled CNTs in random 

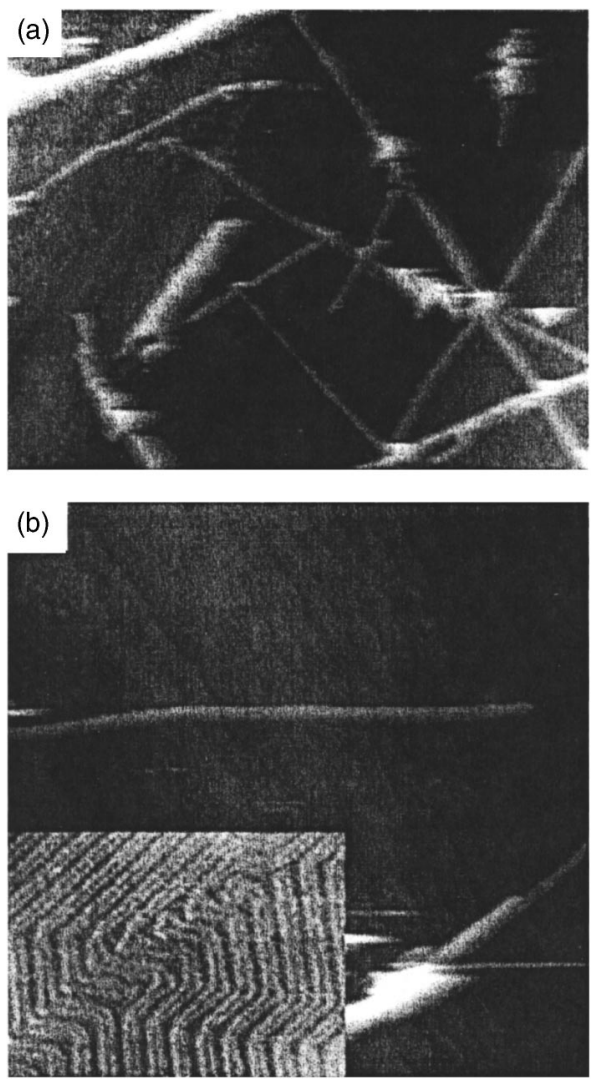

FIG. 1. Typical STM images of CNTs on the Au(111) surface. (a) $300 \times 250 \mathrm{~nm}^{2}$. (b) $220 \times 220 \mathrm{~nm}^{2}$. Inset shows the herringbone reconstruction of a terrace of the $\mathrm{Au}(111)$ surface near CNTs.

distribution. As shown in the inset of Fig. 1(b), we were able to observe the well-known herringbone reconstructions on some parts of the sample, even after these consecutive UHV-Ar environment-UHV procedures. Occasionally, we were able to observe CNT junctions with native defects. All the measurements were done with our home-built low temperature STM, ${ }^{13}$ which can be operated at 5 or $80 \mathrm{~K}$.

\section{RESULTS AND DISCUSSION}

An atomically resolved STM image of a CNT is shown in Fig. 2(a). Most surface area of the CNTs turned out to be atomically clean and well ordered, implying that the CNT surface is chemically stable and inert. We performed a STS measurement with the CNT in Fig. 2(a) and the result is shown in Fig. 2(b). Since the tunneling current $(I)$ of an STM reflects the integrated local density of states of the sample, from the Fermi level to the sample bias voltage $V$, the value of $d I / d V$ is approximately proportional to the local density of state at this energy level. ${ }^{14}[(V / I) d I / d V$ is often used to normalize the bias-dependent tunneling probability.] The LDOS of a CNT can be spatially resolved by taking a $d I / d V$ spectra at selected positions with regular intervals along the center axis of CNT at a fixed tunneling gap or a constant tunneling current. In the present experiment, the STM tip was repeatedly scanned over the topmost line on the same CNTs, leaving the reference current of feedback fixed at 0.5 (a)
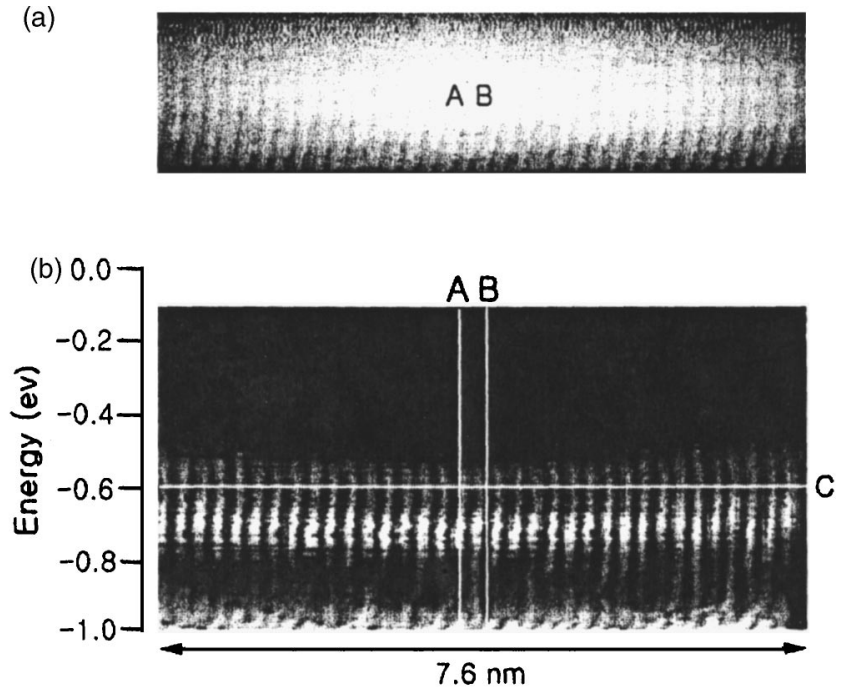

(c)

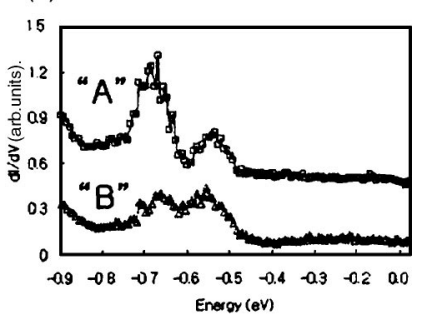

(d)

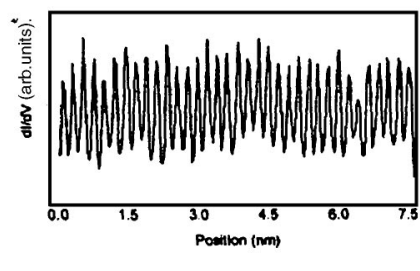

FIG. 2. (a) An STM image of a CNT. The image length is 7.6 $\mathrm{nm}$. The sample bias voltage is $-0.5 \mathrm{~V}$. (b) Spatially resolved LDOS of the CNT. (c) Cross section of (b) along the vertical lines of "A" and "B." (d) Cross section of (b) along the horizontal line of "C."

$\mathrm{nA}$, and changing the bias voltage by a small increment for each line scan. The map of $d I / d V$ was obtained by a lock-in technique with a modulation signal of $\sim 20 \mathrm{mV}$ and $\sim 3 \mathrm{kHz}$ as a function of both the position along the CNT axis and the sample bias voltage. It is presented as a two-dimensional image shown in Fig. 2(b), in which brightness is proportional to the LDOS. Figures 2(c) and 2(d) are the cross sections of Fig. 2(b) along the lines labeled as "A," "B," and "C." The vertical cross sections roughly correspond to the LDOS at the positions "A" and "B" in the STM image of Fig. 2(a). The position "A" is a bright point in the STM image, while the position " $\mathrm{B}$ " is a dark point in the middle of two adjacent bright points. Both LDOS curves show energy gaps, meaning that the measured CNT is semiconducting. The LDOS curves also show two peaks known as van Hove singularity (VHS) peaks, which originate from the one dimensionality of CNTs. ${ }^{1}$ The amplitudes of the two VHS peaks are about the same in the LDOS at the position "B," but not at the position "A." It shows that the spatial distribution of electronic states strongly depends on energy levels, reflecting electronic wave functions of the CNT. Spatial variation is also visible in the horizontal cross section along "C" which shows periodic oscillation with the period of $\sim 2.2 \AA$, the atomic lattice constant. Our LDOS map shows that the spatial distribution of each electronic state can be the atomically resolved in the CNTs. 
(a)
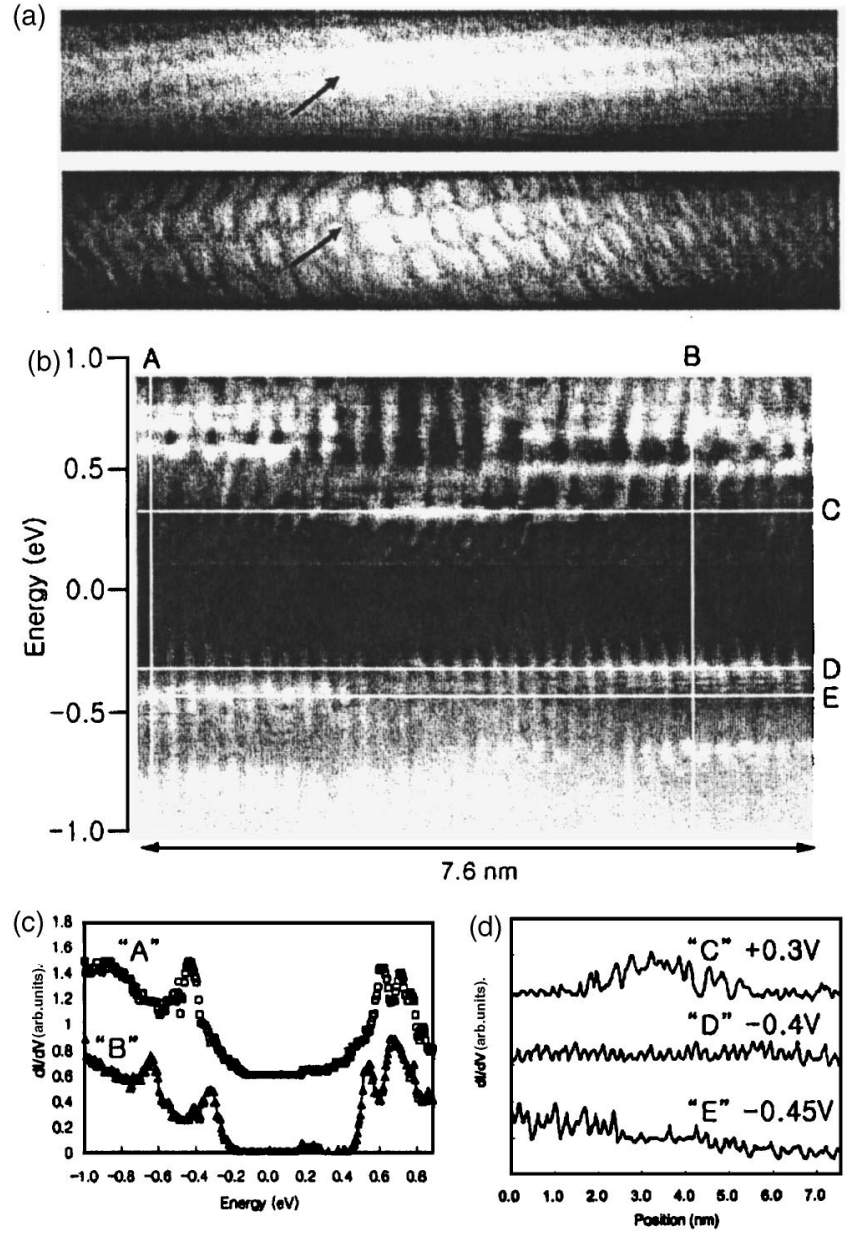

FIG. 3. (a) STM image of a CNT. The image length is $7.6 \mathrm{~nm}$. The sample bias voltage for the upper image is $-0.3 \mathrm{~V}$ and that for the lower image is $0.5 \mathrm{~V}$. (b) Spatially resolved LDOS of the CNT. (c) Cross section of (b) along the vertical lines of "A" and "B." (d) Cross section of (b) along the horizontal lines of "C," "D," and "E."

Figure 3(a) shows bias-dependant STM images taken at another CNT. A defect is visible in the image, as pointed out by arrows in both images. The nature of this defect is not clear in these images. It could be an impurity adsorbate, structural imperfection, or a junction structure connecting two types of CNTs. On the basis of STS data, which we will show later, and the images with other bias voltages, we were able to confirm that the defect is a junction. In the image taken at $+0.5 \mathrm{~V}$, the pattern of the surface corrugation is very different from other bias voltages; the defective region reveals a pattern over a longer period, very similar to the $(\sqrt{3} \times \sqrt{3}) R 30^{\circ}$ structure observed near the defects on graphite surfaces. ${ }^{15}$ As we will discuss in the STS results, this pattern is caused by interface states.

A spatially resolved STS map for the CNT is shown in Fig. 3(b) and the vertical cross sections of it, along the lines labeled as "A" and "B," are shown in Fig. 3(c). The LDOS curves in Fig. 3(c) show different band gaps. The band gaps were measured to be 0.9 and $0.8 \mathrm{eV}$ from the measured VHS peaks, and their chiral indices were estimated to be $(15,2)$ and $(19,3)$, respectively, from the corresponding STM images and spectroscopic features. If the band gaps are deter-

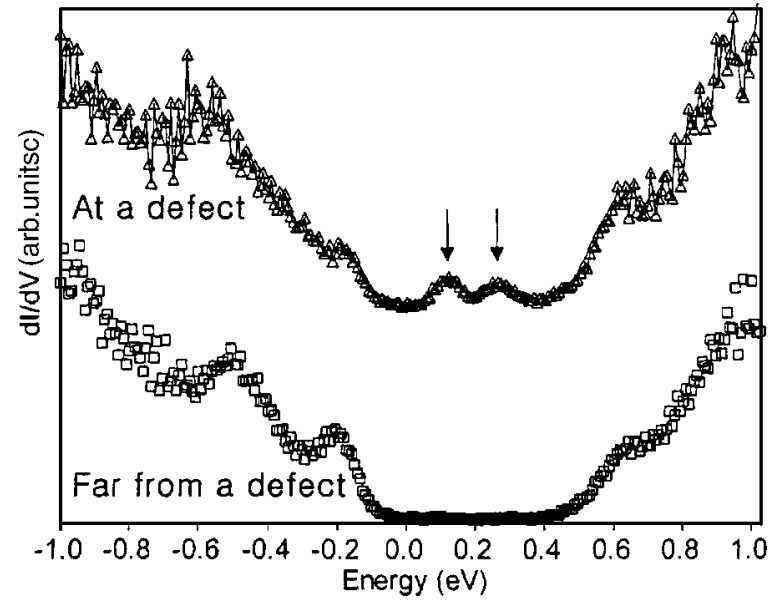

FIG. 4. LDOS data for a CNT with a defect. Upper data was taken at the defect, while lower data was taken far from the defect.

mined from the edges, they are smaller by $\sim 0.2 \mathrm{eV}$. The measured band gaps are larger than the theoretically expected values for given tube diameters. Such broadening of the energy scale appears quite often when band edges are measured with STS. Since the periodic vertical pattern of the STS map reflects the electronic wave functions of CNT, it explains the atomic corrugation observed in the topographic images in Fig. 3(a); the elongated periodicity in the lower image of Fig. 3(a) agrees with that of the vertical lines around the defect at positive bias voltages in Fig. 3(b).

The most prominent feature in Fig. 3(b) is that there is a localized state at the energy of $+0.3 \mathrm{eV}$. Since this energy level is a little below the conduction band edges of both side CNTs, the localized state is a donerlike state. The donerlike state can be understood in terms of Hückel's rule. ${ }^{16}$ In cyclic $\pi$-electron systems, $4 n+2$ ( $n$ : positive integer) number of $\pi$ electrons are more stable than other numbers of $\pi$ electrons (namely, $4 n, 4 n+1,4 n+3$ ). For $n=1$, cyclic rings with six carbon atoms are more stable than those with five and seven carbon atoms, and thus a hexagon structure is more stable than a pentagon or a heptagon. A heptagon will try to give up an electron to its neighbors, and so it plays the role of a donor in a semiconducting CNTs. Similarly, pentagon structures work as an acceptor. It is known that the Stone-Wales transformation is one of the most probable kinetic mechanisms for the defect formation of CNTs, simultaneously producing pentagon-heptagon pairs. ${ }^{7}$ When the defect is composed of pentagon-heptagon pairs, it is expected to be in donerlike and acceptorlike states at the same time. In Fig. 3(b), there is no obvious localized state near the valence band edge. A possibility is that the acceptorlike state is in resonance with the valence band edge state and may not be resolved in the LDOS measurement.

In the experiments with other CNTs, we observed acceptorlike states, as well as doner-like states, around defects. An example is shown in Fig. 4. The two LDOS data are measured from the same CNT at different positions (near and far from a defect). The LDOS data taken far $(\sim 5 \mathrm{~nm})$ from the defect shows typical spectra with a band gap for semiconducting CNTs. The LDOS data taken at the defect show more or less the same spectral shape, except that there are two 
more states, indicated by arrows in Fig. 4. The two states are obviously in the band gap. The higher energy state is donerlike and the lower energy state is acceptorlike. The amplitudes of the two states are about the same, indirectly supporting the possible scenario for a pentagon-heptagon pair defect.

When a junction is formed between two different semiconductors, there is band bending near an interface. The band bending spreads within the charge screening lengths of semiconductors. In our CNT junction, the charge screening lengths can be estimated by measuring the spatial distribution of the interface states. Figure 3(d) is the horizontal cross sections of (b) along the lines labeled as "C," "D," and "E." Since there are no visible peak states at the bias voltage of $-0.4 \mathrm{~V}$, the $d I / d V$ signal in "D" keeps oscillating with an atomic lattice constant. At the bias voltage of $+0.3 \mathrm{~V}$ in " $\mathrm{C}$," the interface state shows a peak at the junction and decays into the left and right CNTs, in convolution with atomic corrugation. For an interface state, the imaginary part of the wave vector is proportional to $\left|E_{b}-\varepsilon_{d}\right|^{1 / 2}$, where $\left|E_{b}-\varepsilon_{d}\right|$ is the difference between the conduction (or valence) band edge and the energy of interface state. Thus, the interface state decays faster to the left than to the right. The screening lengths are estimated to be $\sim 2 \mathrm{~nm}$ to the left CNT and $\sim 3 \mathrm{~nm}$ to the right CNT.

Another feature we see in Fig 3(b) is that the VHS peaks of the left CNT leak into the right CNT, and vice versa. This is clearly visible in the cross section [Fig. 3(d), "E"]. The amplitude of the VHS peak gradually decreases as the measurement position moves across the junction. It does not vanish at the junction, but keeps decreasing even after crossing.
Our S-S CNT junction is a model system for a 1D semiconductor junction. The observed electronic leak is made possible by a low-dimensional effect. Due to the reduced dimension, the charge screening in 1D is less efficient than in bulk structures, allowing the leak of the electronic states at a junction. The electronic leak was observed only within the measured screening lengths.

\section{SUMMARY}

The electronic structures of semiconducting CNTs are spatially resolved. There are spatial oscillations in the electronic density of states in the period of atomic lattice. In an S-S CNT junction, we found a localized interface state. The interface state in the energy gap is donerlike, which possibly originats from a heptagonal atomic structure. The interface state shows unequal decay lengths to the two CNTs, and the decay length was measured to be a few nanometers, longer than in a M-M CNT junction. We observed the electronic leak of the van Hove singularity peaks across a junction, implying inefficient screening in a 1D structure.

\section{ACKNOWLEDGMENTS}

The authors acknowledge financial support by the Ministry of Science and Technology of Korea (National Creative Research Initiatives and National R\&D Project for NanoScience and Technology), by the Ministry of Commerce, Industry and Energy of Korea (Nano-Standard Project), and by Korea Research Foundation (KRF-2003-015-C00208).
${ }^{1}$ M. S. Dresselhaus, G. Dresselhaus, and Ph. Avouris, Carbon Nanotubes: Synthesis, Structures, Properties, and Application (Springer-Verlag, New York, 2001).

${ }^{2}$ C. Dekker, Phys. Today 52, 22 (1999).

${ }^{3}$ Z. Yao, H. W. Ch. Postma, L. Balents, and C. Dekker, Nature (London) 402, 273 (1999).

${ }^{4}$ M. Ouyang, J.-L. Huang, C. L. Cheung, and C. M. Lieber, Science 291, 97 (2001).

${ }^{5}$ J. Lee, H. Kim, S.-J. Kahng, G. Kim, Y.-W. Son, J. Ihm, H. Kato, Z. W. Wang, T. Okazaki, H. Shinohara, and Y. Kuk, Nature (London) 415, 1005 (2002).

${ }^{6}$ C. Zhou, J. Kong, E. Yenilmez, and H. Dai, Science 290, 1552 (2000).

${ }^{7}$ V. Meunier, P. Senet, and Ph. Lambin, Phys. Rev. B 60, 7792 (1999).

${ }^{8}$ D. Orlikowski, M. B. Nardelli, J. Bernholc, and C. Roland, Phys.
Rev. B 61, 14194 (2000).

${ }^{9}$ G. W. Ho, A. T. S. Wee, and J. Lin, Appl. Phys. Lett. 79, 260 (2001).

${ }^{10}$ A. Zangwill, Physics at Surfaces (Cambridge University Press, Cambridge, 1988).

${ }^{11}$ P. Kim, T. W. Odom, J.-L. Huang, and C. M. Lieber, Phys. Rev. Lett. 82, 1225 (1999).

${ }^{12}$ L. C. Venema, J. W. Janssen, M. R. Buitelaar, J. W. G. Wildoer, S. G. Lemay, L. P. Kouwenhoven, and C. Dekker, Phys. Rev. B 62, 5238 (2000).

${ }^{13}$ J.-Y. Park, Ph. D. thesis, Seoul National University (2000).

${ }^{14}$ J. Tersoff and D. R. Hamann, Phys. Rev. B 31, 805 (1985).

${ }^{15}$ J. Xhie et al., Phys. Rev. B 43, 8917 (1991).

${ }^{16}$ T. W. G. Solomons, Organic Chemistry, 6th ed. (Wiley, New York, 1996). 